\title{
Triple Connected Complementary Tree Domination Number of a Graph
}

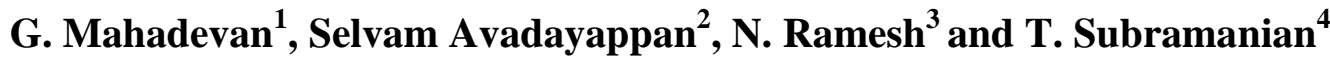 \\ ${ }^{1}$ Dept. of Mathematics, Gandhigram Rural Institute Deemed University, \\ Gandhigram - 624 302, Tamilnadu, India \\ Current address: Department of Mathematics, Anna University: \\ Tirunelveli Region, Tirunelveli - 627 007, Tamilnadu, India \\ gmaha2003@yahoo.co.in \\ ${ }^{2}$ Dept.of Mathematics, VHNSN College \\ Virudhunagar - 626 001, Tamilnadu, India \\ selvam_avadayappan@yahoo.co.in \\ ${ }^{3}$ Udaya School of Engineering, Udaya Nagar \\ Vellamodi, Kanyakumari - 629 204, Tamilnadu, India \\ nrameshnair@yahoo.com \\ ${ }^{4}$ Dept. of Mathematics, Anna University: \\ Tirunelveli Region, Tirunelveli - 627 007, Tamilnadu, India \\ tsubramanian001@gmail.com
}

Copyright (C) 2013 G. Mahadevan et al. This is an open access article distributed under the Creative Commons Attribution License, which permits unrestricted use, distribution, and reproduction in any medium, provided the original work is properly cited.

\begin{abstract}
The concept of triple connected graphs with real life application was introduced in [14] by considering the existence of a path containing any three vertices of a graph G. In [4], G. Mahadevan et. al., introduced triple connected domination number of a graph. A subset $S$ of $V$ of a nontrivial connected graph $G$ is said to be triple connected dominating set, if $S$ is a dominating set and the induced sub graph $\langle S\rangle$ is triple connected. The minimum cardinality taken over all triple connected dominating sets is called the triple connected domination number of $\mathrm{G}$ and is denoted by $\gamma_{t c}(G)$. A subset $S$ of $V$ of a nontrivial graph connected graph $G$ is said to be a complementary tree dominating set, if $S$ is a dominating set and the induced sub graph $\langle V-S\rangle$ is a tree. The minimum cardinality taken over all
\end{abstract}


complementary tree dominating sets is called the complementary tree domination number of $\mathrm{G}$ and is denoted by $\gamma_{c t d}(G)$. In this paper we introduce a new domination parameter, called triple connected complementary tree domination number of a graph. A subset $S$ of $V$ of a nontrivial connected graph $G$ is said to be triple connected complementary tree dominating set, if $S$ is a triple connected dominating set and the induced sub graph $\langle V-S\rangle$ is a tree. The minimum cardinality taken over all triple connected complementary tree dominating sets is called the triple connected complementary tree domination number of $\mathrm{G}$ and is denoted by $\gamma_{t c t}(G)$. We determine this number for some standard graphs and obtain bounds for general graphs. Its relationship with other graph theoretical parameters are also investigated.

\section{Mathematics Subject Classification: 05 C69}

Keywords: Domination Number, Triple connected graph, Triple connected domination number, Triple connected complementary tree domination number.

\section{Introduction}

By a graph we mean a finite, simple, connected and undirected graph $G(V, E)$, where $V$ denotes its vertex set and $E$ its edge set. Unless otherwise stated, the graph $G$ has $p$ vertices and $q$ edges. Degree of a vertex $v$ is denoted by $d(v)$, the maximum degree of a graph $G$ is denoted by $\Delta(G)$. We denote a cycle on $p$ vertices by $C_{p}$, a path on $p$ vertices by $P_{p}$, and a complete graph on $p$ vertices by $K_{p}$. A graph $G$ is connected if any two vertices of $G$ are connected by a path. A maximal connected subgraph of a graph $G$ is called a component of $G$. The number of components of $G$ is denoted by $\omega(G)$. The complement $\bar{G}$ of $G$ is the graph with vertex set $V$ in which two vertices are adjacent if and only if they are not adjacent in G. A tree is a connected acyclic graph. A bipartite graph (or bigraph) is a graph whose vertex set can be divided into two disjoint sets $V_{l}$ and $V_{2}$ such that every edge has one end in $V_{l}$ and another end in $V_{2}$. A complete bipartite graph is a bipartite graph where every vertex of $\mathrm{V}_{1}$ is adjacent to every vertex in $\mathrm{V}_{2}$. The complete bipartite graph with partitions of order $\left|V_{1}\right|=m$ and $\left|V_{2}\right|=n$, is denoted by $K_{m, n}$. A star, denoted by $K_{l, p-1}$ is a tree with one root vertex and $\mathrm{p}-1$ pendant vertices. A bistar, denoted by $B(m, n)$ is the graph obtained by 
joining the root vertices of the stars $K_{l, m}$ and $K_{1, n}$. The friendship graph, denoted by $F_{n}$ can be constructed by identifying $n$ copies of the cycle $C_{3}$ at a common vertex. A wheel graph, denoted by $W_{p}$ is a graph with $p$ vertices, formed by connecting a single vertex to all vertices of $C_{p-1}$. A helm graph, denoted by $H_{n}$ is a graph obtained from the wheel $W_{n}$ by attaching a pendant vertex to each vertex in the outer cycle of $W_{n}$. Corona of two graphs $G_{1}$ and $G_{2}$, denoted by $G_{1} \circ G_{2}$ is the graph obtained by taking one copy of $G_{1}$ and $\left|V_{l}\right|$ copies of $G_{2}\left(\left|V_{l}\right|\right.$ is the number of vertices in $G_{l}$ ) in which $i^{\text {th }}$ vertex of $G_{l}$ is joined to every vertex in the $i^{\text {th }}$ copy of $G_{2}$. If $S$ is a subset of $V$, then $\langle S\rangle$ denotes the vertex induced subgraph of $G$ induced by $S$. The open neighbourhood of a set $S$ of vertices of a graph G, denoted by $N(S)$ is the set of all vertices adjacent to some vertex in $S$ and $N(S) \cup S$ is called the closed neighbourhood of $S$, denoted by N[S]. The diameter of a connected graph is the maximum distance between two vertices in $G$ and is denoted by $\operatorname{diam}(G)$. A cut - vertex (cut edge) of a graph $G$ is a vertex (edge) whose removal increases the number of components. A vertex cut, or separating set of a connected graph $G$ is a set of vertices whose removal results in a disconnected graph. The connectivity or vertex connectivity of a graph $\mathrm{G}$, denoted by $\kappa(G)$ (where $\mathrm{G}$ is not complete) is the size of a smallest vertex cut. A connected subgraph $H$ of a connected graph $G$ is called a $\mathbf{H}$-cut if $\omega(G-H) \geq 2$. The chromatic number of a graph $G$, denoted by $\chi(G)$ is the smallest number of colors needed to colour all the vertices of a graph $G$ in which adjacent vertices receive different colours. For any real number $x,\lfloor x\rfloor$ denotes the largest integer less than or equal to $x$. A Nordhaus-Gaddum-type result is a (tight) lower or upper bound on the sum or product of a parameter of a graph and its complement. Terms not defined here are used in the sense of [11].

A subset $S$ of $V$ is called a dominating set of $G$ if every vertex in $V-S$ is adjacent to at least one vertex in $S$. The domination number $\chi(G)$ of $G$ is the minimum cardinality taken over all dominating sets in $G$. A dominating set $S$ of a connected graph $G$ is said to be a connected dominating set of $G$ if the induced 
sub graph $\langle S\rangle$ is connected. The minimum cardinality taken over all connected dominating sets is the connected domination number and is denoted by $\gamma_{c}$.

Many authors have introduced different types of domination parameters by imposing conditions on the dominating set $[3,16]$. Recently, the concept of triple connected graphs has been introduced by Paulraj Joseph J. et. al.,[14] by considering the existence of a path containing any three vertices of G. They have studied the properties of triple connected graphs and established many results on them. A graph $G$ is said to be triple connected if any three vertices lie on a path in $G$. All paths, cycles, complete graphs and wheels are some standard examples of triple connected graphs. In [4] Mahadevan G. et. al., introduced triple connected domination number of a graph and found many results on them. A subset $S$ of $V$ of a nontrivial connected graph $G$ is said to be triple connected dominating set, if $S$ is a dominating set and the induced sub graph $\langle S\rangle$ is triple connected. The minimum cardinality taken over all triple connected dominating sets is called the triple connected domination number of $\mathrm{G}$ and is denoted by $\gamma_{t c}(G)$. In $[5,6,7,8$, 9, 10] Mahadevan G. et. al., introduced complementary triple connected domination number, paried triple connected domination number, complementary perfect triple connected domination number, triple connected two domination number, restrained triple connected domination number, dom strong triple connected domination number of a graph. A subset $S$ of $V$ of a nontrivial graph connected graph $G$ is said to be a complementary tree dominating set, if $S$ is a dominating set and the induced sub graph $\langle V-S\rangle$ is a tree. The minimum cardinality taken over all complementary tree dominating sets is called the complementary tree domination number of $\mathrm{G}$ and is denoted by $\gamma_{c t d}(G)$. In this paper, we use this idea to develop the concept of triple connected complementary tree dominating set and triple connected complementary tree domination number of a graph.

Theorem 1.1 [14] A connected graph $G$ is not triple connected if and only if there exists a $H$-cut with $\omega(G-H) \geq 3$ such that $\left|V(H) \cap N\left(C_{i}\right)\right|=1$ for at least three components $C_{1}, C_{2}$, and $C_{3}$ of $G-H$. 
Notation 1.2 Let $G$ be a connected graph with $m$ vertices $v_{1}, v_{2}, \ldots ., v_{m}$. The graph obtained from $G$ by attaching $n_{1}$ times a pendant vertex of $P_{l_{1}}$ on the vertex $\mathrm{v}_{1}, n_{2}$ times a pendant vertex of $P_{l_{2}}$ on the vertex $v_{2}$ and so on, is denoted by $G\left(n_{1} P_{l_{1}}\right.$, $\left.n_{2} P_{l_{2}}, n_{3} P_{l_{3}}, \ldots, n_{m} P_{l_{m}}\right)$ where $n_{i}, l_{i} \geq 0$ and $1 \leq i \leq m$.

Example 1.3 Let $v_{1}, v_{2}, v_{3}, v_{4}$, be the vertices of $C_{4}$. The graph $\mathrm{C}_{4}\left(\mathrm{P}_{2}, 2 \mathrm{P}_{2}, 3 \mathrm{P}_{2}, \mathrm{P}_{3}\right)$ is obtained from $\mathrm{C}_{4}$ by attaching 1 time a pendant vertex of $\mathrm{P}_{2}$ on $\mathrm{v}_{1}, 2$ times a pendant vertex of $\mathrm{P}_{2}$ on $\mathrm{v}_{2}, 3$ times a pendant vertex of $\mathrm{P}_{2}$ on $\mathrm{v}_{3}$ and 1 time a pendant vertex of $\mathrm{P}_{3}$ on $\mathrm{v}_{4}$ and is shown in Figure 1.1.

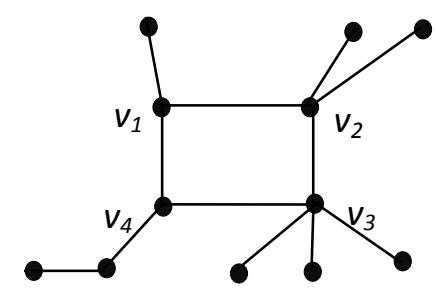

Figure 1.1: $C_{4}\left(P_{2}, 2 P_{2}, 3 P_{2}, P_{3}\right)$

\section{Triple connected complementary tree domination number}

Definition 2.1 A subset $S$ of $V$ of a nontrivial connected graph $G$ is said to be a triple connected complementary tree dominating set, if $S$ is a triple connected dominating set and the induced subgraph $\langle V-S\rangle$ is a tree. The minimum cardinality taken over all triple connected complementary tree dominating sets is called the triple connected complementary tree domination number of $G$ and is denoted by $\gamma_{t c t}(G)$. Any triple connected dominating set with $\gamma_{t c t}$ vertices is called a $\gamma_{t c t}$-set of $G$.

Example 2.2 For the graph $\mathrm{G}_{1}$ in Figure 2.1, $S=\left\{v_{1}, v_{2}, v_{3}\right\}$ forms a $\gamma_{t c t}$-set of $G_{l}$. Hence $\gamma_{\mathrm{tct}}\left(G_{1}\right)=3$.

$G_{1}:$

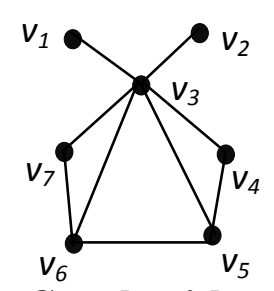

Figure 2.1 : Graph with $\gamma_{\text {tct }}=3$. 
Observation 2.3 Triple connected complementary tree dominating set does not exists for all graphs and if exists, then $\gamma_{\mathrm{tct}}(G) \geq 3$.

Throughout this paper we consider only connected graphs for which triple connected complementary tree dominating set exists.

Observation 2.4 The complement of the triple connected complementary tree dominating set need not be a triple connected complementary tree dominating set.

Observation 2.5 Every triple connected complementary tree dominating set is a dominating set but not conversely.

Observation 2.6 For any connected graph $G, \gamma_{c}(G) \leq \gamma_{t c}(G) \leq \gamma_{t c t}(G)$ and for the cycle $\mathrm{C}_{7}$ the bounds are sharp.

Theorem 2.7 If the induced subgraph of each connected dominating set of $G$ has more than two pendant vertices, then $G$ does not contain a triple connected complementary tree dominating set.

Proof The proof follows from Theorem 1.1.

\section{Exact value for some standard graphs:}

1) For any cycle of order $p \geq 5, \gamma_{t c t}\left(C_{p}\right)=p-2$.

2) For any complete bipartite graph of order $p \geq 5, \gamma_{t c t}\left(K_{m, n}\right)=p-2$.

(where $m, n \geq 2$ and $m+n=p$ ).

3) For any complete graph of order $p \geq 5, \gamma_{t c t}\left(K_{p}\right)=p-2$.

4) For any wheel of order $p \geq 5, \gamma_{t c t}\left(W_{p}\right)=p-2$.

\section{Exact value for some special graphs:}

1) The Moser spindle (also called the Mosers' spindle or Moser graph) is an undirected graph, named after mathematicians Leo Moser and his brother William, with seven vertices and eleven edges given in Figure 2.2. 


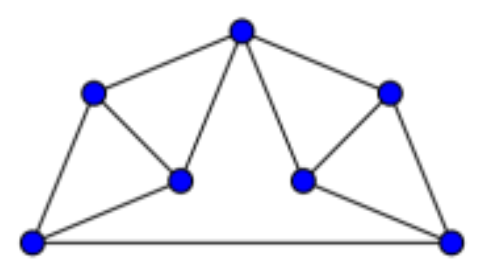

Figure 2.2

For the Moser spindle graph $\mathrm{G}, \gamma_{t c t}(G)=3$.

2) The Wagner graph is a 3-regular graph with 8 vertices and 12 edges given in Figure 2.3.

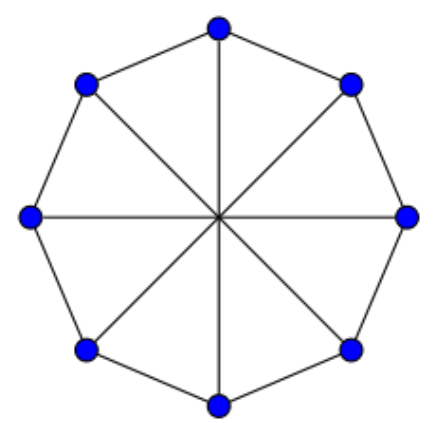

Figure 2.3

For the Wagner graph $\mathrm{G}, \gamma_{t c t}(G)=4$.

3) The Bidiakis cube is a 3-regular graph with 12 vertices and 18 edges given in Figure 2.4.

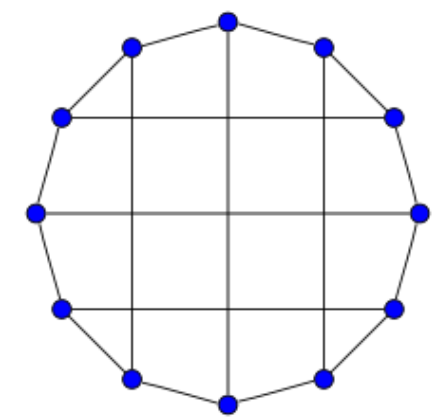

Figure 2.4

For the Bidiakis cube graph $\mathrm{G}, \gamma_{t c t}(G)=8$.

4) The Frucht graph is a 3-regular graph with 12 vertices, 18 edges, and no nontrivial symmetries given in Figure 2.5. 


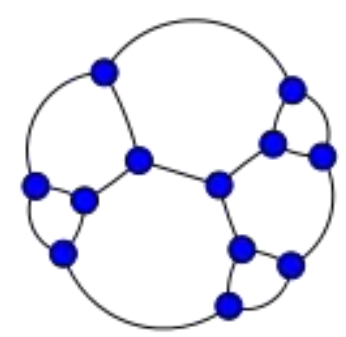

Figure 2.5

For the Frucht graph $\mathrm{G}, \gamma_{t c t}(G)=8$.

Theorem 2.8 For any connected graph $G$ with $p \geq 5$, we have $3 \leq \gamma_{t c t}(G) \leq p-2$ and the bounds are sharp.

Proof The lower and upper bounds follows from Definition 2.1. For $\mathrm{C}_{5}$, the lower bound is attained and for $\mathrm{K}_{6}$ the upper bound is attained.

Theorem 2.9 For a connected graph $G$ with 5 vertices, $\gamma_{t c t}(G)=p-2$ if and only if $G$ is isomorphic to $C_{5}, W_{5}, K_{5}, K_{2,3}, F_{2}, K_{5}-\{e\}, K_{4}\left(P_{2}\right), C_{4}\left(P_{2}\right), C_{3}\left(P_{3}\right), C_{3}\left(2 P_{2}\right)$ or any one of the graphs shown in Figure 2.6.
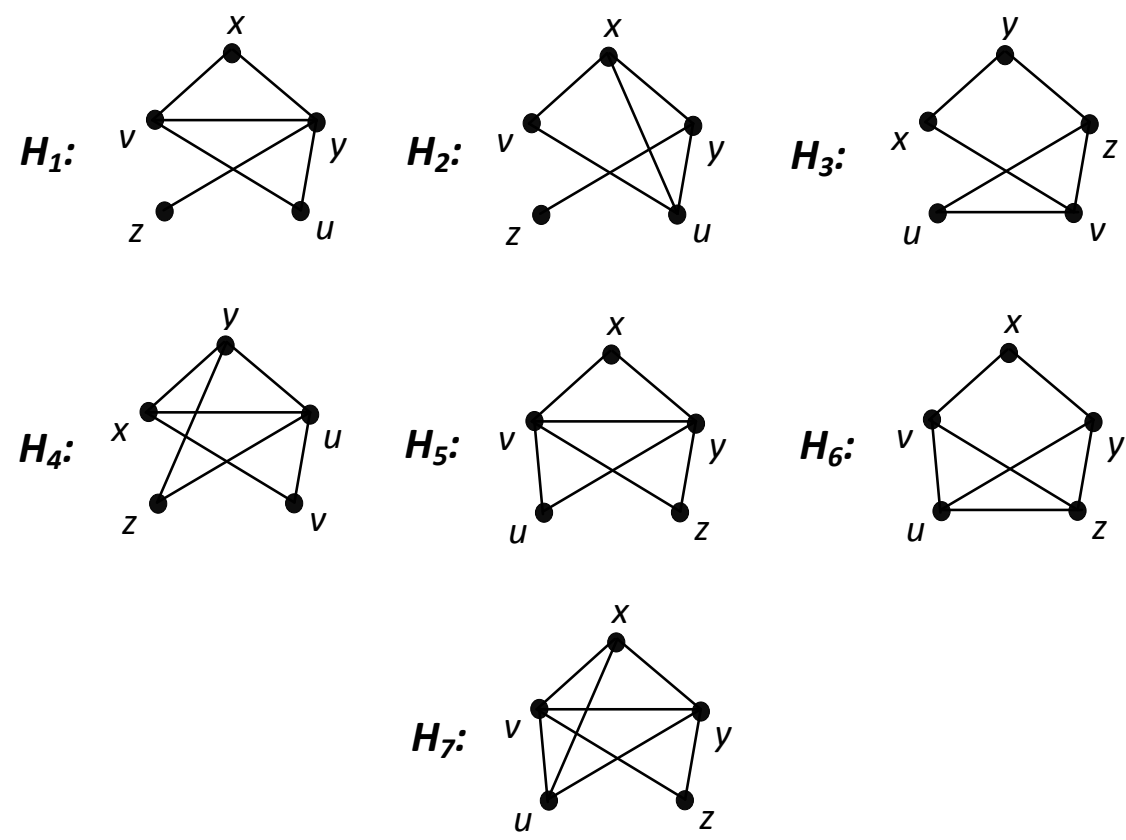

Figure 2.6 : Graphs with $\gamma_{t c t}=p-2$. 
Proof Suppose $G$ is isomorphic to $C_{5}, W_{5}, K_{5}, K_{2,3}, F_{2}, K_{5}-\{e\}, K_{4}\left(P_{2}\right), C_{4}\left(P_{2}\right)$, $C_{3}\left(P_{3}\right), C_{3}\left(2 P_{2}\right)$ or any one of the graphs $H_{1}$ to $H_{7}$ given in Figure 2.2., then clearly $\gamma_{t c t}(G)=p-2$. Conversely, let $G$ be a connected graph with 5 vertices and $\gamma_{t c t}(G)=3$. Let $S=\{x, y, z\}$ be a $\gamma_{t c t}$-set, then clearly $\langle S\rangle=P_{3}$ or $C_{3}$. Let $V-S=V(G)-V(S)=\{u, v\}$, then $\langle V-S\rangle=K_{2}$.

Case (i) $\langle S\rangle=P_{3}=x y z$.

Since $G$ is connected and $\mathrm{S}$ is a $\gamma_{t c t}$-set, there exists a vertex say $x$ (or $z$ ) in $P_{3}$ which is adjacent to $u$ and $v$ in $K_{2}$. Then $S=\{x, y, u\}$ forms a $\gamma_{t c t}$-set of $G$ so that $\gamma_{t c t}(G)=p-2$. If $\quad d(x)=d(y)=2, d(z)=2$, then $G \cong C_{5}$. Since $G$ is connected and $\mathrm{S}$ is a $\gamma_{t c t}$-set, there exists a vertex say y in $P_{3}$ is adjacent to $u$ and $v$

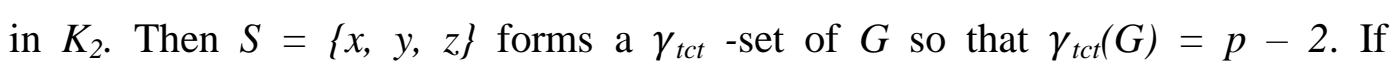
$d(x)=d(z)=1, d(y)=4$, then $G \cong C_{3}\left(2 P_{2}\right)$. Now by increasing the degrees of the vertices, by the above arguments, we have $\mathrm{G} \cong W_{5}, K_{5}, K_{2,3}, K_{5}-\{e\}, K_{4}\left(P_{2}\right)$, $C_{4}\left(P_{2}\right), C_{3}\left(P_{3}\right)$, and $H_{1}$ to $H_{7}$ in Figure 2.2. In all the other cases, no new graph exists.

Case (ii) $\langle S\rangle=C_{3}=x y z x$.

Since $G$ is connected, there exists a vertex say $x$ (or $y, z$ ) in $C_{3}$ is adjacent to $u$ (or $v$ ) in $K_{2}$. Then $S=\{x, u, v\}$ forms a $\gamma_{t c t}$-set of $G$ so that $\gamma_{t c t}(G)=p-2$. If $d(x)=3, d(y)=d(z)=2$, then $G \cong C_{3}\left(P_{3}\right)$. If $d(x)=4, d(y)=d(z)=2$, then $G \cong F_{2}$. In all the other cases, no new graph exists.

Theorem 2.10 Every triple connected complementary tree dominating set must contains all the pendant vertices.

Proof Let $\mathrm{v}$ be a vertex of $\mathrm{G}$ such that $\mathrm{d}(\mathrm{v})=1$ and let $\mathrm{S}$ be a tctd - set of $\mathrm{G}$. If $\mathrm{v}$ is not in $\mathrm{S}$, then a vertex adjacent to $\mathrm{v}$ must be in $\mathrm{S}$ and hence $\langle\mathrm{V}-\mathrm{S}\rangle$ is disconnected, which is a contradiction.

Corollary 2.11 If $\mathrm{G}$ is a graph with $\mathrm{m}$ pendant vertices, then $\gamma_{t c t}(G) \geq m$. 
Proof The proof is directly follows from Theorem 2.10.

The Nordhaus - Gaddum type result is given below:

Theorem 2.12 Let $G$ be a graph such that $G$ and $\bar{G}$ have no isolates of order $p \geq 5$.

Then (i) $\gamma_{t c t}(G)+\gamma_{t c t}(\bar{G}) \leq 2(p-2)$

(ii) $\gamma_{t c t}(G) \cdot \gamma_{t c t}(\bar{G}) \leq 2(p-2)^{2}$ and the bound is sharp.

Proof The bound directly follows from Theorem 2.8. For the cycle $C_{7}$, $\gamma_{t c}(G)+\gamma_{t c}(\bar{G})=2(p-2)$ and for $\mathrm{K}_{5}, \gamma_{t c t}(G) \cdot \gamma_{t c t}(\bar{G}) \leq 2(p-2)^{2}$.

\section{Relation with Other Graph Theoretical Parameters}

Theorem 3.1 For any connected graph $G$ with $p \geq 5$ vertices, $\gamma_{t c t}(G)+\kappa(G) \leq 2 p-3$ and the bound is sharp if and only if $G \cong K_{p}$.

Proof Let $G$ be a connected graph with $p \geq 5$ vertices. We know that $\kappa(G) \leq p-1$ and by Theorem 2.8, $\gamma_{t c t}(G) \leq p-2$. Hence $\gamma_{t c t}(G)+\kappa(G) \leq 2 p-3$. Suppose $G$ is isomorphic to $K_{p}$. Then clearly $\gamma_{t c t}(G)+\kappa(G)=2 p-3$. Conversely, Let $\gamma_{t c t}(G)+\kappa(G)=2 p-3$. This is possible only if $\gamma_{t c t}(G)=p-2$ and $\kappa(G)=p-1$. But $\kappa(G)=p-1$, and so $G \cong K_{p}$.

Theorem 3.2 For any connected graph $G$ with $p \geq 5$ vertices, $\gamma_{\mathrm{tct}}(\mathrm{G})+\chi(\mathrm{G}) \leq 2 p-2$ and the bound is sharp if and only if $G \cong K_{p}$.

Proof Let $G$ be a connected graph with $p \geq 5$ vertices. We know that $\chi(G) \leq p$ and by Theorem 2.8, $\gamma_{t c t}(G) \leq p-2$. Hence $\gamma_{t c t}(G)+\chi(G) \leq 2 p-2$. Suppose $G$ is isomorphic to $K_{p}$. Then clearly $\gamma_{t c t}(G)+\chi(G)=2 p-2$. Conversely, let $\gamma_{t c t}(G)+\chi(G)=2 p-2$. This is possible only if $\gamma_{t c t}(G)=p-2$ and $\chi(G)=p$. Since $\chi(G)=p, G$ is isomorphic to $K_{p}$. 
Theorem 3.3 For any connected graph $G$ with $p \geq 5$ vertices, $\gamma_{t c t}(G)+\Delta(G) \leq 2 p-3$ and the bound is sharp.

Proof Let $G$ be a connected graph with $p \geq 5$ vertices. We know that $\Delta(G) \leq p-1$ and by Theorem 2.8, $\gamma_{t c t}(G) \leq p-2$. Hence $\gamma_{t c t}(G)+\Delta(G) \leq 2 p-3$. For $\mathrm{K}_{8}$, the bound is sharp.

\section{References}

[1] E. A. Nordhaus and, J. W. Gaddum, On complementary graphs, Amer. Math. Monthly, 63 (1956), 175-177.

[2] E. J. Cokayne and, S. T. Hedetniemi, Total domination in graphs, Networks, Vol.10 (1980), 211-219.

[3] E. Sampathkumar and, H. B. Walikar, The connected domination number of a graph, J.Math. Phys. Sci., 13 (6) (1979), 607-613.

[4] G. Mahadevan, A. Selvam, J. Paulraj Joseph and, T. Subramanian, Triple connected domination number of a graph, International Journal of Mathematical Combinatorics, Vol.3 (2012), 93-104.

[5] G. Mahadevan, A. Selvam, J. Paulraj Joseph, B. Ayisha and, T. Subramanian, Complementary triple connected domination number of a graph, Accepted for publication in Advances and Applications in Discrete Mathematics, (2012).

[6] G. Mahadevan, A. Selvam, A. Mydeen bibi and, T. Subramanian, Complementary perfect triple connected domination number of a graph, International Journal of Engineering Research and Application, Vol.2, Issue 5 (2012) , 260-265.

[7] G. Mahadevan, A. Selvam, A. Nagarajan, A. Rajeswari and, T. Subramanian, Paired Triple connected domination number of a graph, International Journal of Computational Engineering Research, Vol. 2, Issue 5 (2012), 1333-1338.

[8] G. Mahadevan, A. Selvam, B. Ayisha, and, T. Subramanian, Triple connected two domination number of a graph, International Journal of Computational Engineering Research Vol. 2, Issue 6 (2012), 101-104.

[9] G. Mahadevan, A. Selvam, V. G. Bhagavathi Ammal and, T. Subramanian, Restrained triple connected domination number of a graph, International Journal of Engineering Research and Application, Vol. 2, Issue 6 (2012), 225-229. 
[10] G. Mahadevan, A. Selvam, M. Hajmeeral and, T. Subramanian, Dom strong triple connected domination number of a graph, American Journal of Mathematics and Mathematical Sciences, Vol. 1, Issue 2 (2012), 29-37.

[11] J. A. Bondy and U. S. R. Murty, Graph Theory, Springer, 2008.

[12] J. Paulraj Joseph and, S. Arumugam, Domination and connectivity in graphs, International Journal of Management Systems, 8 (3) (1992), 233-236.

[13] J. Paulraj Joseph and, S. Arumugam, Domination and coloring in graphs, International Journal of Management Systems, 8 (1) (1997), 37-44.

[14] J. Paulraj Joseph, M. K. Angel Jebitha, P. Chithra Devi and, G. Sudhana, Triple connected graphs, Indian Journal of Mathematics and Mathematical Sciences,Vol. 8, No.1 (2012), 61-75.

[15] J. Paulraj Joseph and, G. Mahadevan, On complementary perfect domination number of a graph, Acta Ciencia Indica, Vol. XXXI M, No. 2. (2006), 847-853.

[16] T. W. Haynes, S. T. Hedetniemi and, P. J. Slater, Domination in graphs, Advanced Topics, Marcel Dekker, New York (1998).

[17] T. W. Haynes, S. T. Hedetniemi and, P. J. Slater, Fundamentals of domination in graphs, Marcel Dekker, New York (1998).

Received: January 11, 2013 